\title{
Non-fluoroscopic radiofrequency catheter ablation of right- and left-sided ventricular arrhythmias
}

\author{
Grzegorz Karkowski ${ }^{1}$, Marcin Kuniewiczz,2, Edward Koźluk², Tomasz Chyży ${ }^{1}$, Andrzej Ząbek ${ }^{1}$, Michał Dusza², \\ Jacek Lelakowski ${ }^{1}$
}

${ }^{1}$ Institute of Cardiology, John Paul II Hospital, Jagiellonian University Medical College, Krakow, Poland

${ }^{2}$ Jagiellonian University Medical College, Krakow, Poland

${ }^{3} 1^{\text {st }}$ Chair and Department of Cardiology, Medical University of Warsaw, Warsaw, Poland

Adv Interv Cardiol 2020; 16, 3 (61): 321-329

DOI: https://doi.org/10.5114/aic.2020.99268

\begin{abstract}
Introduction: Radiofrequency catheter ablation (RFCA) is an important method of treatment of ventricular arrhythmias (VAs). In the majority of RFCA, fluoroscopy is used, exposing patients and medical staff to all related side effects. Current experience of non-fluoroscopic (NF)-RFCA in VAs from the left side is limited.

Aim: Analysis of safety and effectiveness of NF-RFCA of VAs from left- and right-sided cardiac chambers.

Material and methods: From 2014 to 2018, a group of 128 patients who underwent RFCA of VAs were retrospectively divided into two groups: NF-RFCA and fluoroscopic (F)-RFCA. Patients in each group were then subsequently subdivided into two groups based on VAs localization - left- (LS-Va) and right-sided (RS-Va) VAs. In all patients the CARTO Biosense Webster mapping system was used.

Results: In group 1 (NF-RFCA $n=88) 66(75 \%)$ patients underwent RFCA of RS-Va and $22(25 \%)$ of LS-Va. Early success was achieved in $89.8 \%(n=79)$ and long term success in $81.8 \%(n=72)$. In group 2 (F-RFCA $n=40) 19$ patients $(47.5 \%)$ had RFCA of RSVa and $21(52.5 \%)$ patients of LS-Va. Acute procedural success rate was $80 \%(n=32)$ and long-term success $72.5 \%(n=29)$. There were $4(4.6 \%)$ perioperative complications in NF-RFCA and 2 (5\%) in F-RFCA. Success rate, procedure time and complications were not significantly different between groups and subgroups in follow-up.

Conclusions: NF-RFCA in VAs from the right and left cardiac chamber is safe and equally effective as F-RFCA, and it should be implemented as often as possible for protection of patients and electrophysiology staff.
\end{abstract}

Key words: radiofrequency catheter ablation, ventricular arrhythmias, non-fluoroscopic, zero fluoroscopy.

Su m m a ry

This publication focuses on a continually evolving subject - radiofrequency catheter ablation without the use of fluoroscopy. In our study, non-fluoroscopic catheter ablations were performed in both the right and left cardiac chamber. In the publication, we show that this method is effective and safe in the short and long-term observation.

\section{Introduction}

The importance of radiofrequency catheter ablation (RFCA) of ventricular arrhythmias (VAs) is increasing in everyday clinical practice, as well as in the guidelines of cardiological societies [1]. This is related to the high effectiveness and safety of RFCA [2-4]. The majority of VAs are premature ventricular contractions (PVCS) and non-sustained ventricular tachycardia (nsVT), which mainly affect patients with no structural heart disease. PVCs can be highly symptomatic or cause PVC-induced cardiomyopa- thy with decrease of systolic heart function [5]. Treatment of VAs relies on pharmacological therapy (anti-arrhythmic drugs, AAD) or RFCA. RFCA fluoroscopy (ionizing radiation) is commonly used for navigation reasons, which affects the patient and staff due to all associated complications. The use of individual operator shields, shields between the X-ray tube and the operator, and various $X$-ray settings (collimators, optimal angles, optimal intensity settings, etc.) may only reduce, but not eliminate $X$-rays from the electrophysiology (EP) lab. Nowadays, de-

\section{Corresponding author:}

Marcin Kuniewicz PhD, Institute of Cardiology, John Paul II Hospital, Jagiellonian University Medical College, Krakow, Poland, phone: +48 606921 841, e-mail: kuniewiczm@gmail.com

Received: 25.05.2020, accepted: 13.07.2020. 
velopments of 3-dimensional electroanatomical mapping systems (3D-EMS) can help operators to eliminate X-ray usage during RFCA practically in every cardiac chamber [6-8]. Current experience of non-fluoroscopic (NF)-RFCA has shown safety and efficacy, but not many publications reaffirm this strategy of NF-RFCA in VAs from left and right-sided cardiac chambers [2, 3, 6, 9].

\section{Aim}

Analysis of safety, effectiveness and procedural time of non-fluoroscopic RFCA of ventricular arrhythmias from left- and right-sided cardiac chambers.

\section{Material and methods}

From 2014 to 2018, a group of 128 patients who underwent RFCA of ventricular arrhythmias - PVCs - were retrospectively analyzed and divided into two groups: NF-RFCA and fluoroscopic (F)-RFCA groups. In each group, the patients were then subdivided into 2 groups based on PVC localization (Figure 1). Subgroup 1 - ventricular arrhythmia from the right-sided cardiac chamber (RS-Va), which included the ventricular outflow tract (RVOT), and the right ventricle (RV), mainly the region of the tricuspid valve/atrioventricular node. Subgroup 2 - ventricular arrhythmia from the left-sided cardiac chamber (LS-Va), which included the aortic bulb, aortic cusps, left ventricular outflow tract (LVOT) and left ventricle (LV) (out of LVOT). Structural heart disease was defined as ischemic or nonischemic systolic heart failure (EF < 40), previous myocardial infarction $(\mathrm{MI})$, revascularization or significant (moderate or severe) valvular disease.

If pre-procedural examinations and tests (echocardiography, stress test, coronarography or MRI) excluded any structural heart disease, idiopathic VAs was diagnosed. All patients provided a signed statement of consent to undergo the procedure.

\section{Mapping and ablation protocol}

The ablations were performed using the CARTO electroanatomic mapping system (Biosense Webster Johnson \& Johnson) in all included patients.
During the RFCA of RS-VAs, single or double femoral vein puncture was performed. LS-VAs RFCA was performed from single femoral artery access. In some cases, both right and left access was performed.

Ablation catheter electrode was positioned in the right atrium, using anteroposterior (AP) and right-lateral $(\mathrm{RL})$ projections in the CARTO mapping system. Based on the anatomy and electrophysiological signals, the electrode was introduced through the tricuspid valve. The signal from the bundle of His was marked on the CARTO map and activation mapping of RV/RVOT arrhythmia (point by point or fast anatomical map - FAM) was performed (Figures $2 \mathrm{~A}$ and $3 \mathrm{~A}$ ). In the case of PVCS from left ventricle or aortic bulb, CARTO FAM and activation map of the aortic bulb and aortic cusps was performed. The contact force sensor was calibrated in the descending aorta and precise wall touch-ups in sinuses of Valsalva were done with $20 \mathrm{~g}$ maximum. After precise FAM of the ascending aorta was finished the operator crossws the aortic valve with I shape electrode curve. In LV/LVOT (point by point or FAM) mapping was performed (Figure 2 B). The pace-mapping method was always used to confirm the optimal ablation spot if stimulation capture was possible.

PVCS RFCA was performed with irrigated electrodes (energy: 20-40 W, flow: $15-30 \mathrm{ml} / \mathrm{min}$ ), in some cases (from 2016) electrodes had a contact force sensor SmartTouch. All cases were performed (or supported) by operators experienced in F/NF-RFCA.

The early (short-term) efficacy of RFCA was defined as no recurrent arrhythmia (PVCS) after 15 min after the last RF application (Figures $3 \mathrm{~B}, \mathrm{C}$ ). The minimum follow-up period was 12 months. Long-term efficacy was defined as a significant arrhythmia reduction (> $80 \%$ reduction of initial arrhythmia amount) after a healing period (after 3 months) in repeated $24 \mathrm{~h}$ ECG monitoring (every 6-12 months). All necessary medical follow-up data (12-lead ECG, 24-hour Holter ECG, repeat ablation information) were obtained from outpatient medical records.

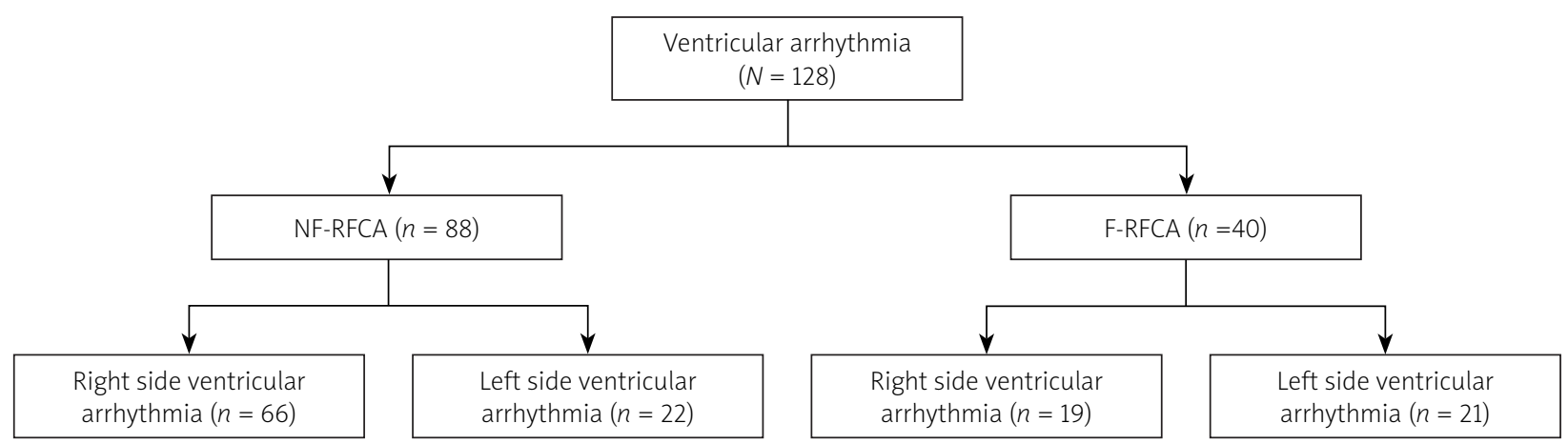

Figure 1. Division of the patients with VAs into groups and subgroups based on type of RFCA (NF or F) and arrhythmia localization

VAs - ventricular arrhythmias, RFCA - radiofrequency catheter ablation, NF-non-fluoroscopic, F-fluoroscopic. 


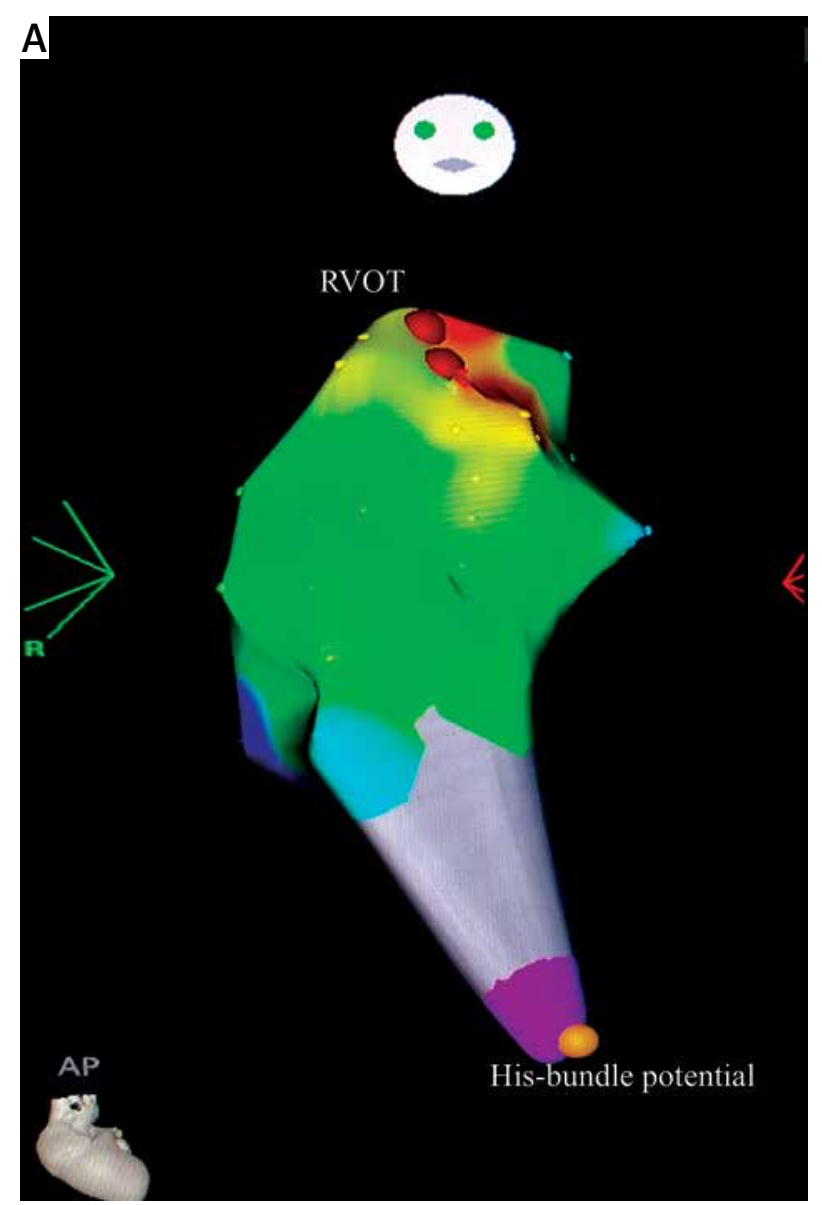

A

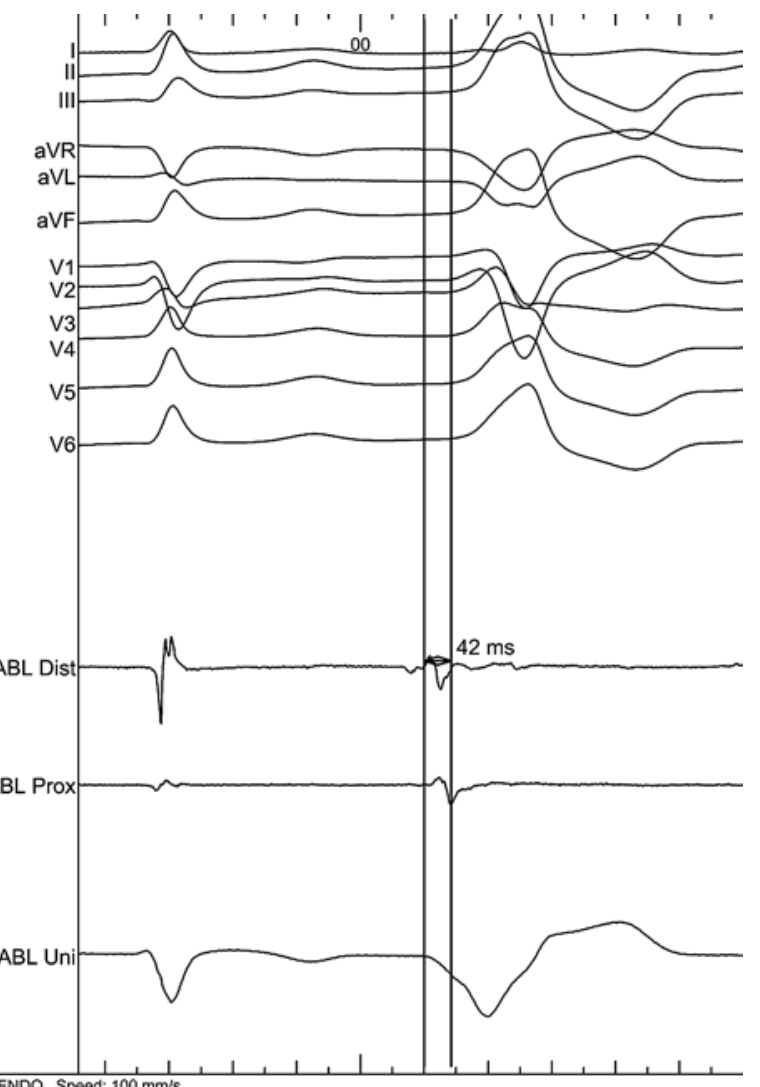

B

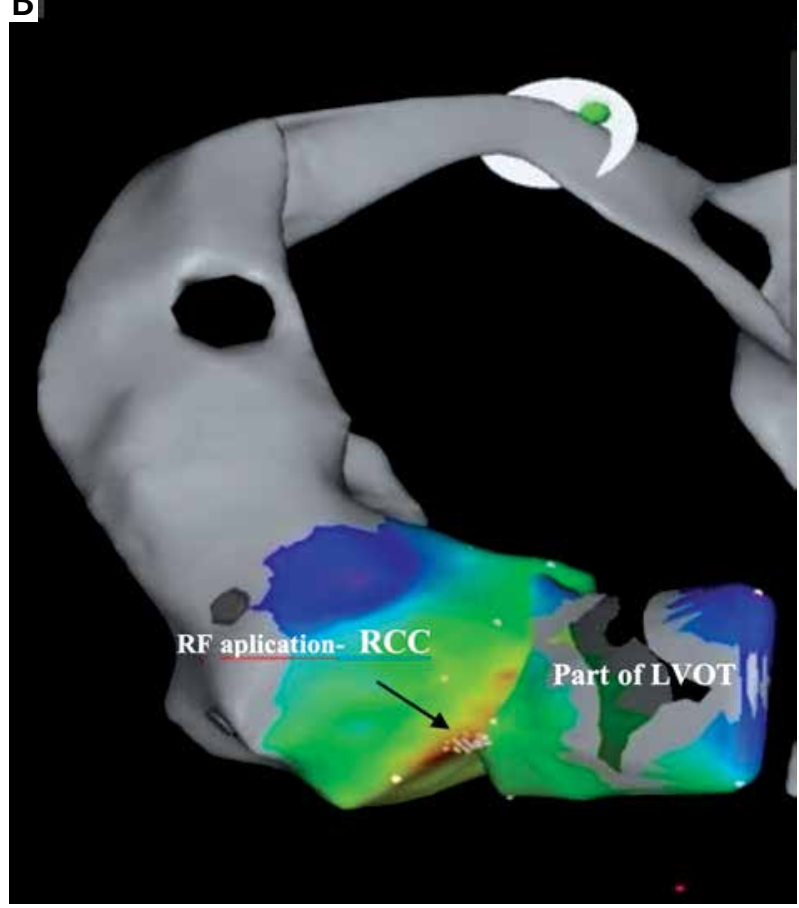

Figure 2. A - CARTO activation map of premature ventricular contractions from RVOT. Yellow dot - localization of His bundle, red dots - RF applications at the earliest ectopic beats activation. B - Right coronary cusp of the aortic valve, red dots and black arrow - RF application site

Figure 3. A - Endocardial potential from RF application point 


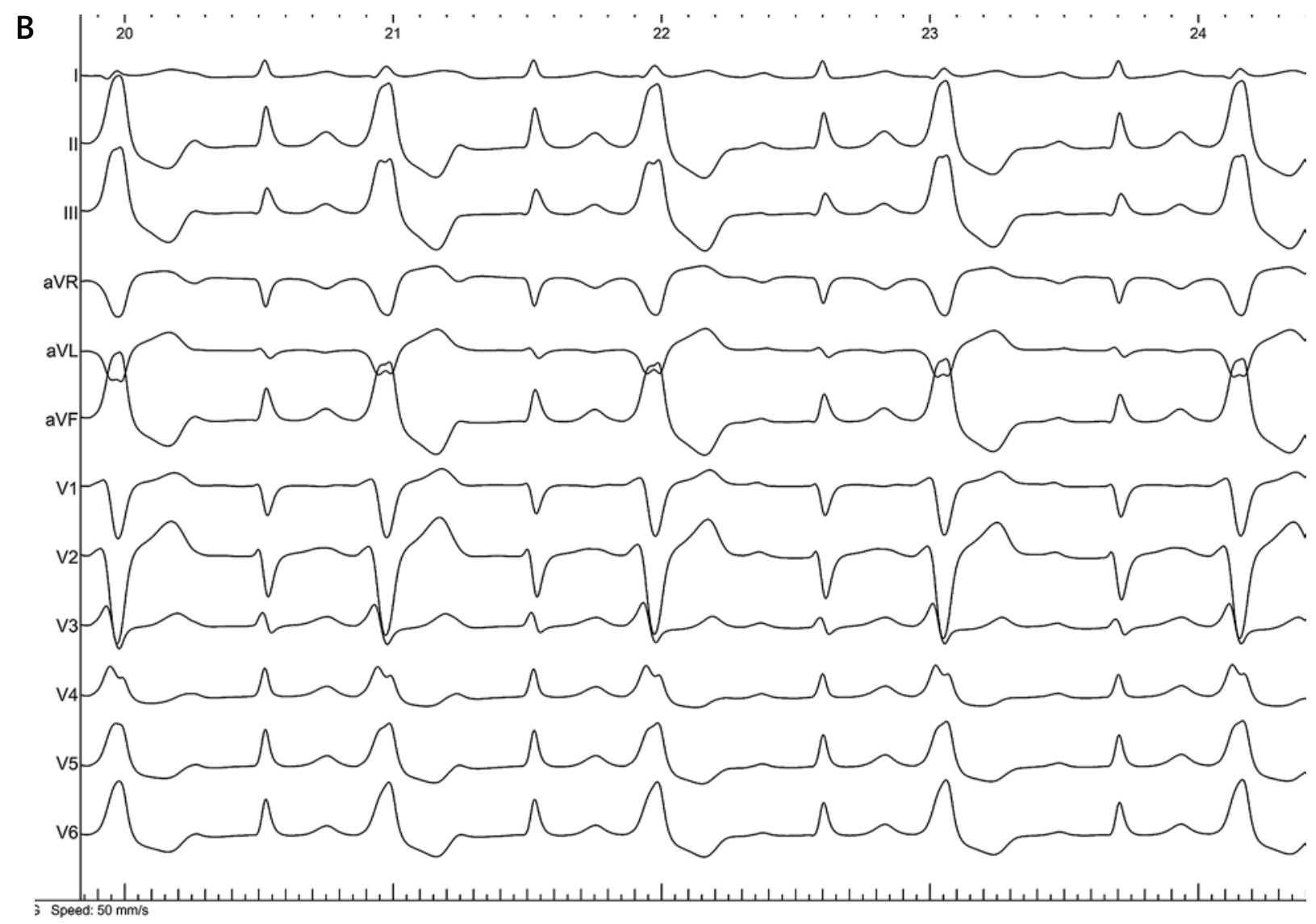

C

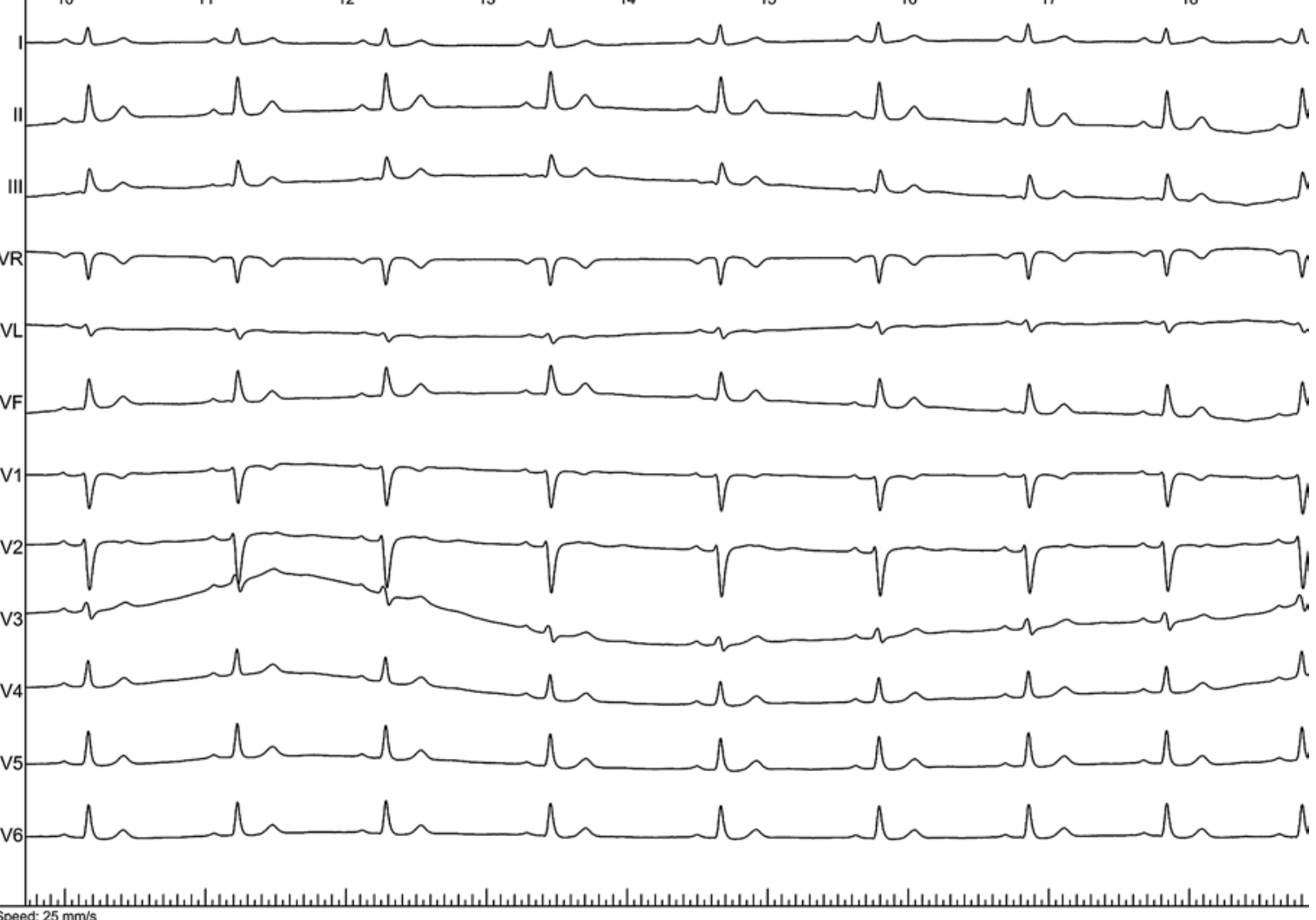

Figure 3. Cont. B - 12-lead ECG before RF application, C - 12-lead ECG after RF applications 
Table I. Patient characteristics

\begin{tabular}{|c|c|c|c|c|}
\hline Parameter & $\begin{array}{c}\text { Total } \\
(N=128)\end{array}$ & $\begin{array}{l}\text { NF-RFCA } \\
(n=88) \\
\end{array}$ & $\begin{array}{c}\text { F-RFCA } \\
(n=40) \\
\end{array}$ & $P$-value \\
\hline Age of patients [years], Me (Q1-Q3) & $48(35.0-61.5)$ & $42.0(33.0-58.0)$ & $61.0(52.5-68.0)$ & $<0.001$ \\
\hline Female, $n(\%)$ & $68(53.1)$ & $52(59.1)$ & $16(40.0)$ & 0.045 \\
\hline Hypertension, $n$ (\%) & $51(39.8)$ & $25(28.4)$ & $26(65.0)$ & $<0.001$ \\
\hline $\mathrm{DM}, n(\%)$ & $11(8.6)$ & $4(4.5)$ & $7(17.5)$ & 0.037 \\
\hline Presence of CIED, $n$ (\%) & $3(2.3)$ & $1(1.1)$ & $2(5.0)$ & 0.230 \\
\hline History of AF, $n(\%)$ & $12(9.4)$ & $6(6.8)$ & $6(15.0)$ & 0.252 \\
\hline History of CAD, $n(\%)$ & $26(20.3)$ & $10(11.4)$ & $16(40.0)$ & $<0.001$ \\
\hline Systolic heart failure, $n$ (\%) & $9(7.0)$ & $4(4.5)$ & $5(12.5)$ & 0.137 \\
\hline BMI 25-30 kg/m²,n (\%) & $15(11.7)$ & $7(7.9)$ & $8(20.0)$ & 0.095 \\
\hline $\mathrm{BMI}>30 \mathrm{~kg} / \mathrm{m}^{2}, n(\%)$ & $8(6.2)$ & $2(2.3)$ & $6(15.0)$ & 0.011 \\
\hline Number of antiarrhythmic drugs before ablation (1), Me (Q1-Q3) & $0.5(0.0-1.0)$ & $0.0(0.0-1.0)$ & $1.0(0.5-1.0)$ & 0.003 \\
\hline Fluoroscopy time [min], Me (Q1-Q3) & $0.0(0.0-1.4)$ & $0.0(0.0-0.0)$ & $4.3(1.6-11.6)$ & $<0.001$ \\
\hline
\end{tabular}

AF-atrial fibrillation, BMI - body mass index, CAD - coronary artery disease, CIED - cardiac implantable electronic device, DM - diabetes mellitus, NF-RFCA - nonfluoroscopic radiofrequency catheter ablation, F-RFCA - fluoroscopic radiofrequency catheter ablation.

\section{4-h Holter monitoring}

24-h Holter monitoring was performed in all patients. Examinations were performed according to the Polish Cardiac Society [10]. The 24-h Holter monitoring was performed prior to ablation and was repeated 3 months after RF ablation and, after that, at least once a year during a follow-up.

\section{Echocardiography}

Transthoracic echocardiography (TTE) was performed in all patients. Examinations were performed with a Vivid S6 (GE Healthcare, U.S.A.) device, according to the European Association for Cardiovascular Imaging (EACVI) and Polish guidelines at the time of patient enrolment [11].

The institutional ethics committee approved the study protocol and written informed consent was obtained from all patients for the use of their anonymous data in the present publication. The study protocol conformed to the ethical guidelines of the 1975 Declaration of Helsinki.

\section{Statistical analysis}

Data were analyzed using IBM SPSS Statistics Version 25.0 software (IBM Corp, Armonk, New York, United States). Continuous variables are expressed as mean (SD), and median (interquartile range (Q1-Q3)) if not normally distributed. The Shapiro-Wilk test was used to assess the normality of continuous variables. Mean values were compared using Student's $t$-test, with Mann-Whitney tests used for continuous variables when a normal distribution was not present. For comparisons of 4 independent groups of continuous variables, we used a one-way analysis of variance (ANOVA), and for variables with non-normal distribution we used a nonparametric Kruskal-Wallis test. For multiple comparisons between 2 groups, the Bonferroni correction was applied.

The categorical variables were presented as the number of observations in each category and the percentage of observations in this category out of all observations. The $\chi^{2}$ test and the Fisher exact test were performed to compare categorical variables.

All statistical tests were 2-tailed, and a $p$-value of 0.05 was considered statistically significant.

\section{Results}

\section{Baseline characteristics}

In group 1 (NF-RFCA) there were 88 patients and in group 2 (F-RFCA) 40 patients. The baseline patient characteristics are detailed in Table I.

\section{Group characteristics}

In group 1 (NF-RFCA) 66 (75\%) patients had RFCA of RS-VAs and 22 (25\%) patients of LS-VAs. Early success was $89.8 \%(n=79)$ and long term success $81.8 \%(n=72)$. Mean procedural duration was $80.0 \mathrm{~min}$ (65.0--107.5). Mean follow-up time was 47.5 (34.0-64.0) months. Arrhythmia localization in RS-VAs was mainly from RVOT $-83.5 \%(n=55)$ and in this subgroup long-term success was $87.5 \%$. In the rest of the patients, RS-VAs originates from the tricuspid valve (mostly parahisian) region $16.5 \%(n=11)$ with long term efficiency $54.5 \%$. 
LS-VAs were localized in $36 \%(n=8)$ in the aortic bulb and aortic cusps, in $32 \%(n=7)$ in the LVOT, and in $32 \%$ $(n=7)$ in the LV.

In group 2 (F-RFCA) 19 (47.5\%) patients had RFCA of RS-VAs and 21 (52.5\%) patients of LS-VAs. Early success was $80 \%(n=32)$ and long term success $72.5 \%(n=29)$. Mean follow-up time was 46.0 (34.0-70.0) months. In RS-VAs arrhythmia originates in $90 \%(n=15)$ from the RVOT with long term success $80 \%$ and from out of the RVOT region (mainly parahisian) in $10 \%(n=4)$ with long term success rate $50 \%$. In LV-VAs RF ablation was per- formed in $19 \%(n=4)$ in the aortic bulb and aortic cusps, in $38 \%(n=8)$ in the LVOT and in $43 \%(n=9)$ in the LV. Detailed rates of success are presented in Table II.

\section{NF-RFCA vs. F-RFCA overall}

Early and long term efficiency RFCA was not significantly different between groups and subgroups (Table II). Procedural duration differs (borderline) between groups 1 and $2(p=0.041)$. In subgroups analysis, procedural duration was significantly longer in LS-VAs in F-RFCA compared to RS-VAs in both F and NF- RFCA overall.

Table II. Follow-up details

\begin{tabular}{|c|c|c|c|c|c|}
\hline \multirow[t]{2}{*}{ Parameter } & \multicolumn{2}{|c|}{$\begin{array}{l}\text { NF-RFCA } \\
(n=88)\end{array}$} & \multicolumn{2}{|c|}{$\begin{array}{l}\text { F-RFCA } \\
(n=40)\end{array}$} & \multirow[t]{2}{*}{$P$-value } \\
\hline & $\begin{array}{l}\text { Right side ventricu- } \\
\text { lar arrhythmia } \\
\text { (group A) } \\
(n=66)\end{array}$ & $\begin{array}{l}\text { Left side ventricular } \\
\text { arrhythmia } \\
\text { (group B) } \\
(n=22)\end{array}$ & $\begin{array}{l}\text { Right side ventricu- } \\
\text { lar arrhythmia } \\
\text { (group C) } \\
(n=19)\end{array}$ & $\begin{array}{l}\text { Left side ventricular } \\
\text { arrhythmia } \\
\text { (group D) } \\
(n=21)\end{array}$ & \\
\hline \multirow{2}{*}{$\begin{array}{l}\text { Age of patients [years], } \\
\text { Me (Q1-Q3) }\end{array}$} & \multicolumn{2}{|c|}{$42.0(33.0-58.0)$} & \multicolumn{2}{|c|}{$61.0(52.5-68.0)$} & $<0.001$ \\
\hline & $40.5(32.0-49.0)$ & $59.0(38.0-72.0)$ & $60.0(40.0-66.0)$ & $63.0(59.0-69.0)$ & $\begin{array}{c}<0.001 \\
0.018^{\#} \\
0.024^{\# \#} \\
<0.001^{\# \# \#} \\
>0.99^{*} \\
0.564^{\star *} \\
>0.99^{\star * \star}\end{array}$ \\
\hline \multirow[t]{2}{*}{ Female, $n(\%)$} & \multicolumn{2}{|c|}{$52(59.1)$} & \multicolumn{2}{|c|}{$16(40.0)$} & 0.045 \\
\hline & $42(63.6)$ & $10(45.5)$ & $7(36.8)$ & $9(42.9)$ & 0.095 \\
\hline \multirow{2}{*}{$\begin{array}{l}\text { Duration of procedure } \\
\text { [min], Me (Q1-Q3) }\end{array}$} & \multicolumn{2}{|c|}{$80.0(65.0-107.5)$} & \multicolumn{2}{|c|}{$97.5(77.5-115.0)$} & 0.041 \\
\hline & $77.5(60.0-103.0)$ & $90.0(70.0-120.0)$ & $80.0(60.0-95.0)$ & $110.0(100.0-120.0)$ & $\begin{array}{c}<0.001 \\
0.660^{\#} \\
>0.99^{\# \#} \\
<0.001^{\# \# \#} \\
0.99^{*} \\
0.564^{\star \star} \\
0.009^{\star \star \star}\end{array}$ \\
\hline \multirow[t]{2}{*}{ Complications, $n$ (\%) } & \multicolumn{2}{|c|}{$4(4.6)$} & \multicolumn{2}{|c|}{$2(5.0)$} & $>0.99$ \\
\hline & $2(3.0)$ & $2(9.1)$ & $0(0.0)$ & $2(9.5)$ & 0.257 \\
\hline \multirow[t]{2}{*}{ Early success, $n(\%)$} & \multicolumn{2}{|c|}{$79(89.8)$} & \multicolumn{2}{|c|}{$32(80.0)$} & 0.131 \\
\hline & $59(89.4)$ & $20(90.9)$ & $15(78.9)$ & $17(81.0)$ & 0.468 \\
\hline \multirow[t]{2}{*}{ Long term success, $n$ (\%) } & \multicolumn{2}{|c|}{$72(81.8)$} & \multicolumn{2}{|c|}{$29(72.5)$} & 0.231 \\
\hline & $54(81.8)$ & $18(81.8)$ & $13(68.4)$ & $16(76.2)$ & 0.604 \\
\hline \multirow{2}{*}{$\begin{array}{l}\text { Duration of follow-up } \\
\text { [months], Me (Q1-Q3) }\end{array}$} & \multicolumn{2}{|c|}{$48.6 \pm 16.7 ; 47.5(34.0-64.0)$} & \multicolumn{2}{|c|}{$49.3 \pm 16.3 ; 46.0(34.0-70.0)$} & 0.767 \\
\hline & $52.0(34.5-65.5)$ & $42.0(28.5-47.5)$ & $58.0(34.0-70.0)$ & $46.0(34.0-46.0)$ & $\begin{array}{c}0.037 \\
0.400^{\#} \\
>0.99^{\# \#} \\
0.703^{\# \# \#} \\
0.086^{*} \\
>0.99^{\star *} \\
0.151^{\star * \star}\end{array}$ \\
\hline
\end{tabular}

Data are presented as median (Q1-Q3) or number (\%). "P-value between group $A$ and $B, \# p$-value between group $A$ and $C$, \#\#\#-value between group $A$ and $D$, ${ }^{*} p$-value between group $B$ and $C,{ }^{* *} p$-value between group $B$ and $D,{ }^{* * *} p$-value between group $C$ and $D$. NF-RFCA - non-fluoroscopic radiofrequency catheter ablation, F-RFCA - fluoroscopic radiofrequency catheter ablation. 
Patients with RS-VAs in the NF-RFCA group were significantly younger compared to other subgroups; this relationship was not observed comparing LS-VAs in NF-RFCA.

\section{Complications}

There were $4(4.6 \%)$ perioperative complications in NF-RFCA. One pseudoaneurysm after femoral artery access - treated by thrombin injection, one first degree of atrioventricular block - with PQ interval $270 \mathrm{~ms}$ after ectopic ventricular ablation from the parahisian region and two episodes of pericarditis after ablation in the aortic root and one case in the tricuspid valve region. In one case, pericarditis required pharmacotherapy, in the second after about 2 weeks, the patient required pericardiocentesis because of the symptomatic pericardial effusion. In the F-RFCA group, there were 2 (5\%) perioperative complications - hematomas, which did not require blood transfusion. There was no difference in complication rate between the groups and subgroups $(p>0.99)$ (Table II).

\section{NF-RFCA vs. F-RFCA procedural time}

In general, there was no difference in procedural time between subgroups. Comparing time over right side procedures with fluoroscopy and without (groups A and C), the medians are almost equal $(77.5 \mathrm{~min}$ vs. $80.0 \mathrm{~min}$, $p=0.99$ ). In left side procedures (groups $B$ and $D$ ), there was no statistical difference $(90.0 \mathrm{~min}$ vs. $110.0 \mathrm{~min}$, $p=0.54)$. Comparing right vs left side in each procedure type in NF-RFCA (groups A and B) the times in left side procedures were longer, although without significance (77.5 min vs. $90.0 \mathrm{~min}$ ), while in the right side procedures with fluoroscopy usage the difference was significant (80.0 $\mathrm{min}$ vs. $110.0 \mathrm{~min}, p=0.009$ ) (Table II).

\section{Discussion}

RF Catheter ablation has the potential to be the firstline therapy of ventricular arrhythmias. ESC guidelines recommend RFCA of VAs ahead of AAD therapy only in RVOT localization. This localization has a good profile of safety and efficacy for RFCA. In different localizations of VAs, AAD therapy has a higher recommendation class than RFCA. Additionally, RFCA should be performed in experienced centers due to possible complications that are rare but severe (aorta dissection, tamponade or coronary vessel injury) $[1,4]$. Except for symptoms, a major indication for RFCA is PVC-induced cardiomyopathy. A high PVC burden is one of the most important factors that indicate patients' predisposition to cardiomyopathy development. A cut-off point of PVC burden value is not known precisely. In publications, the burden value of PVCs varies, but $24 \%$ has been proposed as having the best sensitivity and specificity for the prediction of cardiomyopathy, but a lower value also has been suggested [12]. Furthermore, PVC-QRS duration > $140 \mathrm{~ms}$, presence of interpolated PVCs, and PVC coupling intervals $\leq 600$ $\mathrm{ms}$ are also associated with the risk of cardiomyopathy development [5, 13, 14].

Recent publications show an overall success rate of RFCA in idiopathic PVCs without distinguishing localization at $84 \%$. In some localizations, RFCA is more effective (RVOT), in others less effective (papillary muscles) [4]. In our study, right-sided VA (out of RVOT), mostly located in the tricuspid region (parahisian), had the lowest efficiency in both NF and F-RFCA, which was mainly due to proximity of the atrioventricular node (AVN) where usage of RF energy was limited. In such cases, patients were referred for cryoablation.

3D-EMS allows one to reduce fluoroscopy over the years, but recent times have brought the development of non-fluoroscopic (NF) technology, which has started to be used more widely in the EP lab, becoming very often the strategy of choice [15]. In Wang's paper [2], prospective, multicenter study, long term efficacy and safety of NF-RFCA in VAs were equal to F-RFCA $n=127$ (84.1\%) Vs. $(n=274)(85.4 \%)$. The author did not distinguish in detail the location of PVCs. The AVATAR Registry and Zhu's paper concerned VAs from aortic cusps (more challenging area). The authors demonstrated that NF-RFCA is effective and safe, the same as F-RFCA, even in such a "risky" area $[3,9]$. The present study also shows the efficacy and safety of NF-RFCA from both right/left-sided VAs. The most sensitive stage of left-sided VAs-RFCA is mapping/ablation of aortic cusps, the ostium of coronary arteries, and crossing the aortic valve. Both recent studies and the present one have shown that it is safe during NF-RFCA, especially when an ablation catheter in which there was a contact force sensor was used [2, 3, 9]. Nevertheless, navigation and ablation inside aortic bulb/ cusps with hemodynamic/cardio-surgery backup should be considered. Optimally, NF-RFCA in the mentioned region should be facilitated by intracardiac echocardiography $[16,17]$. We suggest that operators should start training in NF-RFCA using right-sided VA cases from the very beginning. In addition, in this observation, patients in the NF-RFCA group were younger and had fewer comorbidities. It shows that operators are still choosing fluoroscopy support for suspected anatomic difficulties (e.g. obesity, atherosclerosis).

The safety and efficacy of NF-RFCA have also been confirmed in other arrythmias such as supraventricular tachycardia, atrial flutter and atrial fibrillation and structural VT $[6,7,18]$. Moreover, cryoablation procedures also demonstrated a good profile of NF-catheter ablation as well as "radiation-sensitive" groups of patients such as children and pregnant women [19-22]. Yang's meta-analysis of 10 studies, including 2,261 patients, showed efficacy and safety of NF-RFCA. Unfortunately, it analyzed only a few cases of VAs (max. 15 patients) [6]. 
The procedural time was comparable to F-RFCA in each subgroup with a nonsignificant procedural time increase for F-RFCA. Similar observations with assessment of a learning curve were found in a different publications [16].

Exposure to X-rays among patients and medical staff, due to the development of medical/diagnostic techniques, is constantly increasing. Invasive cardiologists are the most exposed group among all medical professions. Being exposed to X-rays, medical staff are at increased risk of developing neoplasms (leukemia, brain tumors, breast cancer), genetic defects in progeny, and more frequent occurrence of cataracts [23-25]. In addition, many years of using personal radiological protection (such lead aprons) exposes staff to diseases of the osteoarticular system (backache, knee pain) [25]. Heidbuchel summarized valuable tips on limiting the exposure of medical personnel to ionizing radiation in his work [26]. The most important point is the operator's awareness of the harmfulness of radiation and the need to minimize it wherever possible without decreasing procedural safety and efficiency. Monitoring the exposure of personnel to radiation level is very important, but it must be emphasized that there is no "safe" dose at which no harmful effect can be considered in a professional lifetime period.

The main study limitation is a relatively small sample of included patients and single-center set-up. The study exhibited a retrospective form and included no randomization.

\section{Conflict of interest}

The authors declare no conflict of interest.

\section{References}

1. Authors/Task Force M, Priori SG, Blomstrom-Lundqvist C, Mazzanti A, et al. 2015 ESC Guide-lines for the management of patients with ventricular arrhythmias and the prevention of sudden cardiac death: The Task Force for the Management of Patients with Ventricular Arrhythmias and the Pre-vention of Sudden Cardiac Death of the European Society of Cardiology (ESC) Endorsed by: Association for European Paediatric and Congenital Cardiology (AEPC). Eur Heart J 2015; 36: 2793-867.

2. Wang Y, Chen GZ, Yao Y, et al. Ablation of idiopathic ventricular arrhythmia using zero-fluoroscopy approach with equivalent efficacy and less fatigue: a multicenter comparative study. Medicine 2017; 96: e6080.

3. Zhu TY, Liu SR, Chen YY, et al. Zero-fluoroscopy catheter ablation for idiopathic premature ventricular contractions from the aortic sinus cusp. Nan Fang Yi Ke Da Xue Xue Bao 2016; 36: 1105-9.

4. Latchamsetty R, Yokokawa M, Morady F, et al. Multicenter outcomes for catheter ablation of idiopathic premature ventricular complexes. JACC Clin Electrophysiol 2015; 1: 116-23.

5. Panizo JG, Barra S, Mellor G, et al. Premature ventricular complex-induced cardiomyopathy. Arrhythm Electrophysiol Rev 2018; 7: 128-34.

6. Yang L, Sun G, Chen X, et al. Meta-analysis of zero or near-zero fluoroscopy use during ablation of cardiac arrhythmias. Am J Cardiol 2016; 118: 1511-8.
7. Bulava A, Hanis J, Eisenberger M. Catheter ablation of atrial fibrillation using zero-fluoroscopy technique: a randomized trial. Pacing Clin Electrophysiol 2015; 38: 797-806.

8. Haegeli LM, Stutz L, Mohsen M, et al. Feasibility of zero or near zero fluoroscopy during catheter ablation procedures. Cardiol J 2019; 26: 226-32.

9. Styczkiewicz K, Ludwik B, Śledź J, et al. Long-term follow-up and comparison of techniques in radiofrequency ablation of ventricular arrhythmias originating from the aortic cusps (AVATAR Registry). Pol Arch Intern Med 2019; 129: 399-407.

10. Baranowski R, Bieganowska K, Cygankiewicz I, et al. Wytyczne dotyczące wykonywania długotrwałych rejestracji EKG. Stanowisko grupy ekspertów Sekcji Elektrokardiologii Nieinwazyjnej i Telemedycyny Polskiego Towarzystwa Kardiologicznego. Kardiol Pol 2013; 71 (Supl. IX): 225-41.

11. Steeds RP, Garbi M, Cardim N, et al. 2014-2016 EACVI Scientific Documents Committee; 2014-2016 EACVI Scientific Documents Committee. EACVI appropriateness criteria for the use of transthoracic echocardiography in adults: a report of literature and current practice review. Eur Heart J Cardiovasc Imaging 2017; 18: 1191-204.

12. Baman TS, Lange DC, Ilg KJ, et al. Relationship between burden of premature ventricular complexes and left ventricular function. Heart Rhythm 2010; 7: 865-9.

13. Yokokawa M, Kim HM, Good E, et al. Impact of QRS duration of frequent premature ventricular complexes on the development of cardiomyopathy. Heart Rhythm 2012; 9: 1460-4.

14. Olgun $\mathrm{H}$, Yokokawa M, Baman T, et al. The role of interpolation in RVC-induced cardiomyopathy. Heart Rhythm 2011; 8: 1046-9.

15. Marini W, Martin M, Ravanelli D, et al. Extensive use of 3D nonfluoroscopic mapping systems for reducing radiation exposure during catheter ablation procedures: an analysis of 10 years of activity. Biomed Res Int 2019; 2019: 4217076.

16. Rivera S, Vecchio N, Ricapito P, Ayala-Paredes F. Non-fluoroscopic catheter ablation of arrhythmias with origin at the summit of the left ventricle. J Interv Cardiac Electrophysiol 2019; 56: 279-90.

17. Sánchez JM, Yanics MA, Wilson P, et al. Fluoroless catheter ablation in adults: a single center experience. J Intervent Cardiac Electrophysiol 2016; 45: 199-207.

18. Sadek MM, Ramirez FD, Nery PB, et al. Completely nonfluoroscopic catheter ablation of left atrial arrhythmias and ventricular tachycardia. J Cardiovasc Electrophysiol 2019; 30: 78-88.

19. Koźluk E, Rodkiewicz D, Piątkowska A, Opolski G. Safety and efficacy of cryoablation without the use of fluoroscopy. Cardiol J 2018; 25: 327-32.

20. Scaglione M, Ebrille E, Caponi D, et al. Zero-fluoroscopy ablation of accessory pathways in children and adolescents: CARTO3 electroanatomic mapping combined with RF and cryoenergy. Pacing Clin Electrophysiol 2015; 38: 675-81.

21. Akdeniz C, Gul EE, Celik N, et al. Catheter ablation of idiopathic right ventricular arrhythmias in children with limited fluoroscopy. J Interv Cardiac Electrophysiol 2016; 46: 355-60.

22. Koźluk E, Piątkowska A, Kiliszek M, et al. Catheter ablation of cardiac arrhythmias in pregnancy without fluoroscopy: a case control retrospective study. Adv Clin Exp Med 2017; 26: 129-34.

23. Roguin A, Goldstein J, Bar O, Goldstein JA. Brain and neck tumors among physicians performing interventional procedures. Am J Cardiol 2013; 111: 1368-72.

24. Pani A, Giuseppina B, Bonanno C, et al. Predictors of zero X-ray ablation for supraventricular tachycardias in a nationwide mul- 
ticenter experience. Circ Arrhythm Electrophysiol 2018; 11: e005592.

25. Buchanan GL, Chieffo A, Mehilli J, et al. The occupational effects of interventional cardiology: results from the WIN for Safety survey. Eurolntervention 2012; 8: 658-63.

26. Heidbuchel H, Wittkampf FHM, Vano E, et al. Practical ways to reduce radiation dose for patients and staff during device implantations and electrophysiological procedures. Europace 2014; 16: 946-64. 